\title{
On the Importance of Considering Country-specific Aspects on the Online- Market: An Example of Music Recommendation Considering Country- Specific Mainstream
}

\author{
Christine Bauer \\ Johannes Kepler University Linz \\ christine.bauer@jku.at
}

\author{
Markus Schedl \\ Johannes Kepler University Linz \\ markus.schedl@jku.at
}

\begin{abstract}
In the field of music recommender systems, country-specific aspects have received little attention, although it is known that music perception and preferences are shaped by culture; and culture varies across countries.

Based on the LFM-1b dataset (including 53,258 users from 47 countries), we show that there are significant country-specific differences in listeners' music consumption behavior with respect to the most popular artists listened to. Results indicate that, for instance, Finnish users' listening behavior is farther away from the global mainstream, while United States' listeners are close to the global mainstream.

Relying on rating prediction experiments, we tailor recommendations to a user's level of preference for mainstream (defined on a global level and on a country level) and the user's country. Results suggest that, in terms of rating prediction accuracy, a combination of these two filtering strategies works particularly well for users of countries far away from the global mainstream.
\end{abstract}

\section{Introduction}

In the era of digitalization, particularly due to the developments of social platforms, the amount of available and consumable content (e.g., news, videos, movies, music, products) has increased tremendously [40]. This opportunity to access a large amount of content often results in information overload [9] and users require novel mechanisms and strategies to choose from the deep blue sea of content [48]. Thus, recommender systems have become important tools in people's everyday lives and are used in such activities as for shopping [3,33,41] or consuming news [40], movies [35,62], and music $[17,47,50]$.

Recommender systems are meant to assist users in searching, sorting, and filtering the massive amount of online content [38]. This, in turn, helps to decrease the problem of information overload [40].

For instance, access to music recordings was for the longest time restricted to local availability of their physical representations (e.g., CD, DVD, vinyl). Now, music has become easier to access than ever: Users have access to tens of millions of musical recordings using online music platforms such as YouTube, Spotify, or iTunes [48]. Music recommender systems (MRS) have been adopted to assist listeners in navigating through the myriad of available musical works and to provide them with suggestions that may fit their preferences and/or needs [7]. Currently, MRS are important drivers in the music industry and are widely adopted by music platforms [7]. They have also become a significant research topic over the past few years $[17,50]$.

"The success of a music recommender system (RS) depends on its ability to propose the right music, to the right user, at the right moment" [30]. However, this task is extremely complex, as various factors influence a user's music preferences in a given situation. Numerous studies have investigated the relationship between music preferences and, amongst others, demographics $[13,21,29]$, personality traits $[14,43]$, social influences $[10,59]$, user activity [63], or listening habits [51].

Still, most MRS algorithms rely mainly on useritem interactions (in collaborative filtering) or on information about music items (in content-based recommenders) [12,28,34,53,57]. Such information about music items ranges from acoustic features such as rhythm, melody, or timbre $[11,16,17]$, to editorial metadata such as genre or release year [12,36], to usergenerated collaborative data such as annotations via tags $[25,32]$, to annotations gathered via web content mining $[54,60]$.

It was not until a few years ago that context-aware MRS began to receive considerable attention (e.g., [1]). Context-aware systems are systems that are aware of the context that they are used in and/or their users' context and adapt their operations to the current 
context without explicit user intervention [8]. Dey and Abowd [23] define context as "any information that can be used to characterize the situation of an entity", including, for example, location, personality traits, wind chill factor, etc., cf. [8]. Context may be derived from various sources, including a user's active input, data available on the web (e.g., a user's personal information on social media), or from sensors such as, for instance, integrated in every common smartphone (e.g., accelerator). While a few music players allow a user to specify his or her mood or activity to tailor the music recommendations accordingly, to the best of our knowledge, no fully automated context-aware music recommenders have been released to the public yet [53]. Most recommender systems, in research and in practice, largely disregard the variety of context aspects influencing a user's (music) preferences or needs [8].

For instance, there are various country-specific mechanisms that affect a user's music preferences and consumption behavior. Music perceptions vary across cultures [31,39,56,58]. Music preferences are shaped by cultural aspects and vary across countries $[6,15]$. National market structures, including distribution channels, legislation, subsidizing, and local radio airplay are different across countries [26,42,45]. For instance, recently Budzinski and Pannicke [15] found that pop music preferences disconverge rather than converge in European countries. As a result, countryspecific aspects shape users' music preferences and music consumption behavior and also which artists are popular is country-specific.

Still, being part of the entertainment economy, the music market shares the specific nature that there is a high concentration of demands for the most popular items (the head), whereas there is a long tail of less popular items that fulfil niche demands [17]. The implication for MRS is that it is more likely that a random user will like a very popular music item than one of the far less popular items [17,52]. And so popularity-based MRS approaches are widely adopted, in particular to complement other approaches (e.g., $[19,64])$.

However, what most previous approaches to music popularity for MRS share is that they view music popularity from a global perspective and adopt a fraction-based approach [49], which disproportionately privileges the global absolute top hits (the head). This, in turn, leads to lower performance in rating prediction accuracy for global niche consumers in collaborative filtering approaches. For instance, from a global perspective, Finnish users who like the artist "Katariina Hänninen" are niche consumers, whereas given the Finnish artist popularity charts, these users are national top hit consumers.
Against this background, we emphasize that music platforms might benefit from tailoring their recommendation algorithms to target users differently depending on their national culture. The research gap may be summarized as follows: With respect to MRS research, little is known about how the systems could and should integrate country-specific factors in order to provide better recommendations to better satisfy a wide variety of users, i.e., users covering a wider range of must preferences (not only global top hit consumers).

Based on the rating prediction approach commonly employed in collaborative filtering recommender systems [44], we investigate the performance differences (in terms of rating prediction accuracy) realized for users when both their national culture and their global as well as country-specific mainstreamreflection in listening behavior are considered. To this end, we use the LFM-1b dataset [48] of user-generated listening events (based on 53,258 users from 47 countries) from Last.fm. Country is considered a proxy for national culture in the present study. Being aware that the concept of national culture has been criticized for equating culture with nation and leaving aside ethnic aspects [27,37], we emphasize that next to cultural aspects also national market structures contribute to users' music consumption preferences and behavior. Thus, country as proxy seems reasonable for the study at hand.

The work at hand delivers two main contributions: First, we show the existence of considerable countryspecific differences in listening behavior with respect to the degree of deviation from the global mainstream. More specifically, we differentiate between and account for a global versus a country-specific music mainstream measurement, which is a novel asset. We show that (i) there are countries where users' music consumption behavior corresponds to the global mainstream, (ii) there are countries where a countryspecific mainstream has developed in addition to the global mainstream, and (iii) there are countries with many outliers, thus, not having established a clear picture concerning music mainstream consumption behavior. Second, we demonstrate how considering a user's country, which we use as proxy for cultural background (here: national culture), in the personalized music recommendation process can notably improve accuracy of rating prediction, compared to a one-fitsall solution without country information.

The remainder of this paper is structured as follows: In Section 2, we outline the conceptual foundations of our work. Section 3 details the methods and procedures employed in our research. Section 3 reports the results. In the final section, we conclude with a summary and an outlook to future work. 


\section{Conceptual Foundations}

In this section, we first outline the basics of MRS (Section 2.1). Then we discuss related work adopting popularity-based recommendation approaches and describe the essence of the so-called "music mainstreaminess of a user" and its importance for MRS (Section 2.2).

\subsection{Music Recommender Systems}

Recommender systems are software tools and techniques that suggest items that are likely relevant for a particular user to assist the respective user in his or her decision-making process (e.g., what products to buy, what music to listen to or what online news to read) [44]. Recommender systems consist of three key components: users, items, and user-item-matching mechanisms. This general structure also applies for MRS, where users are the listeners and items are the music items (music recordings) [7]. User-itemmatching mechanisms are traditionally based on one of the three main techniques: content-based filtering, collaborative filtering, or hybrid approaches that combine the other two filtering techniques [2]. Using a content-based filtering approach, an MRS recommends items based on a comparison between the content of items (e.g., extracted via audio analysis and/or from keywords or tags about music items) and at least one indication of preference of a user. MRS employing collaborative filtering, in contrast, do not need exogenous information about either items or users; instead, they maintain for each listener a user profile holding either implicit or explicit preference indications (e.g., ratings or other user-item-interactions such as number of listening events). Music items listened to by users with similar preferences and/or listening patterns are then recommended to the target user [44]. Collaborative filtering is a highly researched approach and the most widely adopted in industry [62]. The goal of hybrid approaches is to achieve synergy effects and, thus, provide better recommendations than any filtering technique would supply on its own, while avoiding their limitations and problems, such as cold start (new user and new item problem) or sparsity (typically, very few user-item-interactions are available) [17].

\subsection{Popularity and Mainstreaminess Characteristics for Music Recommendations}

The phenomenon that there is a high concentration of demands for the most popular items, which form the head of the demand distribution, and a long tail of less popular items [17] is coined the "long-tail" economy [4,5]; but also other terms are used to refer to the phenomenon, in particular in the music industry (e.g., the "hit-driven paradigm" [17] or the "long-tail concept" $[17,18])$, using various terms or phrases to refer to the most popular music items (e.g., "hits" [17] or the "short head" [22], the "mainstream music" $[11,17,18]$, etc.).

As it is more likely that a random user will like a very popular item than one of the far less popular items [17,52], popularity-based recommender system approaches are widely adopted; in particular to complement other approaches in cold start situations, for instance, in the music domain (e.g., $[19,64])$, but also in other domains such as news (e.g., [65]), or product recommendation in e-commerce in general (e.g., [3]).

While various ways exist to define and measure popularity (for instance, in terms of sales figures, media coverage, radio air plays, etc.), in the field of MRS, the popularity of a music item is frequently characterized by the total playcount of the respective item, i.e., the number of listening events the item realizes by all listeners in total, cf. [17]. Alternatively, the number of unique listeners of the item, referred to as listener count or listener frequency, can be used [55]. Note that these measures are different from those typically used in other domains, in particular from those used in movie recommendation, where the most frequently used preference elicitation strategy is that of asking users to provide explicit ratings (e.g., on a Likert-type rating scale between 1 and 5).

Considering popularity, music listeners may be described "in terms of the degree to which they prefer music items that are currently popular or rather ignore such trends" [47] which was coined "music mainstreaminess of a user" [47,51]. It describes how strongly a user's music playcounts of artists, albums, or tracks correspond to the respective playcounts of the overall population. A few recent studies $[51,61]$ have shown that leveraging music mainstreaminess in combination with collaborative filtering techniques delivers better results with respect to recommendation accuracy and rating prediction error than pure collaborative filtering approaches alone [51].

Still, a limitation of this early work on user mainstreaminess for MRS is that the authors adopt socalled fraction-based approaches when modeling userspecific mainstreaminess [49]. These approaches quantify mainstreaminess as fractions of the target user's playcounts among the playcounts of the overall population, which disproportionately privileges the absolute top hits (the head). This in turn leads to lower performance when considering the corresponding 
fraction-based user models of mainstreaminess in collaborative filtering approaches. A more recent, and more promising, approach to gauge user mainstreaminess is based on rank-order correlations, i.e., global and user-specific playcounts are converted to ranks and the correlation between the two are computed [49]. In our experiments reported here, we will use this rank-based definition as it has been shown to yield better results than fraction-based approaches [49].

However, previous approaches in the MRS field disregard country-specific difference. Calling on this research gap, we will first show in the next section that what is considered mainstream depends on the selection of a population (in our case, global ${ }^{1}$ versus country-wise) and then demonstrate that considering a user's country for creating music recommendations considerably decreases prediction error compared to a global scope.

\section{Methods}

In the work at hand, we follow a two-step approach. First, we analyze country-specific differences of the distribution of (mainstream) music. We use the publicly available LFM-1b dataset of usergenerated listening events from Last.fm [48] for this investigation. It can be downloaded from a dedicated web page. ${ }^{2}$

Second, in line with common recommender systems evaluation, we perform rating prediction experiments using again the LFM-1b dataset. In particular, we analyze the performance of a state-ofthe-art collaborative filtering recommender when tailoring the recommendations to user groups defined according to their level of mainstreaminess and their cultural background. More specifically, we analyze two ways to define mainstreaminess: on a global level and on a country level. For both scopes, we group users according to their mainstreaminess into three classes (low, medium, and high mainstreaminess).

Section 3.1 describes the sample of the LFM-1b dataset we use in our study. In Section 3.2, we discuss the deployed approach for mainstreaminess measurement. Section 3.3 details the recommendation setup for the rating prediction experiments and outlines the evaluation metrics that we use to assess the performance of recommendations.

\footnotetext{
${ }^{1}$ Note that the global population is in our case the Last.fm users in the dataset of our study, irrespective of country.

${ }^{2}$ http://www.cp.jku.at/datasets/LFM-1b
}

\subsection{Dataset Sample}

We use the LFM-1b dataset [48] that comprises $1,088,161,692$ listening events of 120,322 unique users. The essential part for our analyses is the userartist-playcount matrix (UAM) containing the listening events of 120,175 unique users to 585,095 unique artists. The distribution of artist playcounts resembles a typical long-tail distribution [17]. Since our investigation focuses on country-specific differences, we consider a subset of the LFM-1b dataset, which only includes listening events of users who provided country information. To reduce possible noise and obtain meaningful results, we furthermore only consider countries with at least 100 users. The respective filtering of the dataset results in 53,258 users from 47 countries. In order to perform the evaluation of recommender systems via rating prediction (Section 3.3), we subsequently normalize and scale the playcount values in the UAM to the range $[0,1000]$, for each user individually; higher numbers of playcounts thus indicate a higher preference for the artists by the user.

\subsection{Mainstreaminess Measurement Approach}

We define the artist frequency $A F_{a, u}$ as the sum of listening events to tracks by artist $a$ listened to by user $u$. Accordingly, we define $A F_{a, c}$ as the sum of listening events to tracks by artist $a$ listened to by all users in country $c$. In the following, when not otherwise said, country $c$ is always the country user $u$ originates in. Finally, we define $A F_{a}$ as the total number of listening events to tracks by artist $a$ listened to by the entire population in the dataset, i.e. on a global scale. We compute these user-specific, country-specific, and global artist frequencies over all 585,095 artists in the dataset and represent them as a 585,095-dimensional vector.

We refer to this vector representation of a user's, the country-specific, and the global artist frequencies as preference profile $P P_{u}, P P_{c}$, and $P P_{g}$, respectively. Based on a state-of-the-art measure for mainstreaminess [49], we calculate rank-order correlation according to Kendall's $\tau$, between the global and user-specific preference profiles as well as between the country-specific and user-specific preference profiles, as shown in Equations (1) and (2),

$$
\begin{aligned}
& R_{u, g}=\tau\left(\operatorname{ranks}\left(P P_{u}\right), \operatorname{ranks}\left(P P_{g}\right)\right) \\
& R_{u, c}=\tau\left(\operatorname{ranks}\left(P P_{u}\right), \operatorname{ranks}\left(P P_{c}\right)\right)
\end{aligned}
$$


where $\operatorname{ranks}\left(P P_{u}\right)$ denotes a function that converts the real-valued preference profile of user $u$ to ranks, $\operatorname{ranks}\left(P P_{c}\right)$ accordingly on the country-level (country of user $u)$, and $\operatorname{ranks}\left(P P_{g}\right)$ on the global level, i.e. considering all users. Higher values therefore indicate closer to the (country or global) mainstream, whereas lower ones indicate farther away from the mainstream.

\subsection{Recommendation Setup}

To compare the performance of recommender systems for various user groups (defined by mainstreaminess and scope, i.e., global or country), we apply singular value decomposition (SVD) according to [46] equivalent to probabilistic matrix factorization, to factorize the corresponding UAM and in turn effect rating prediction. In 5-fold cross-validation experiments, we use root mean square error (RMSE) as the performance measure.

To investigate the influence of both, the two mainstreaminess definitions (global vs. countryspecific; Equations (1) and (2)) and mainstreaminess levels on recommendation performance, we then create for each combination of mainstreaminess measure and country subsets of users. More specifically, we split the users in each country into three (almost) equally sized subsets according to their mainstreaminess value: low corresponds to users in the lower 3-quantile (tertile) w.r.t. the respective mainstreaminess definition, mid and high, respectively, to the mid and upper tertile. Then, we conduct the same rating prediction experiment on all users in each country (user set all) and in addition to each subset (user sets high, mid, and low) in each country. This allows for a comparison of a pure mainstreaminess filtering approach (global) versus a combination of mainstreaminess filtering and country filtering (country-specific).

\section{Results}

First, we report on the results on country-specific differences of users' listening behavior concerning music mainstreaminess (Section 4.1). Motivated by the results, we show in Section 4.2 how tailoring to country-specific characteristics of mainstreaminess may yield improved recommendations.

\subsection{Country-specific Differences in Listeners' Music Mainstreaminess}

Our results indicate clearly that there are countryspecific differences in listeners' music mainstreaminess. While the trend is the same when using either artist frequency or listener frequency as a measure of popularity, the differences are more distinctive for listener frequency. As prototypical examples, we visualize the listener frequency distributions for the United States (US), Finland (FI), and Sweden (SE) in the Figures 1-3, respectively.

Figure 1 indicates that listeners in the United States are close to the global mainstream. Finland, in contrast, can be considered a country that does particularly not correspond to the global music mainstream. The observable second line above the main distribution indicates that there exists a distinct Finish mainstream in parallel to the global mainstream (Figure 2). The visualization of listener frequency distribution for Sweden (Figure 3) does not clearly indicate the existence of a Swedish mainstream parallel to the global one. Still, there are a lot of outliers, representing strong deviations in the Swedish users' listening behavior as compared to the global mainstream.

Due to the dominating role of the United States on the global music market, it is not surprising that US users' mainstreaminess reflects the global one. More interesting is the finding that listeners in Finland, a small country with little importance on the global music market, have developed a distinct music culture that is strongly manifested in this country's users' listening behavior. Sweden, in contrast, a country historically known for its prominent success in exporting pop music, shows neither a very strong affinity to global mainstream, nor a tendency towards any strong Swedish mainstream. However, the deviations in country-specific listener frequencies from the global ones are clearly indicated in the visualizations. We therefore want to emphasize that while Finland and Sweden are geographically close, according to our results the music listening habits seem to be largely different in these two countries.

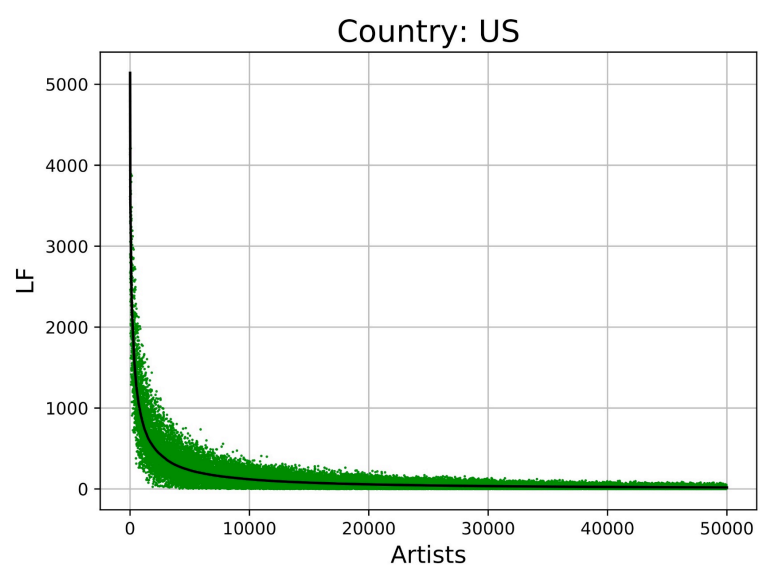

Figure 1. Listener frequency distribution over artists for the United States 


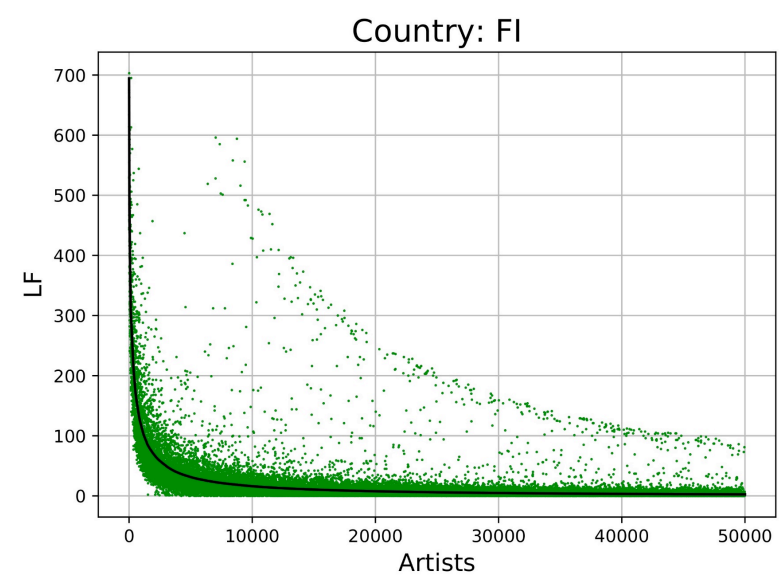

Figure 2. Listener frequency distribution over artists for Finland

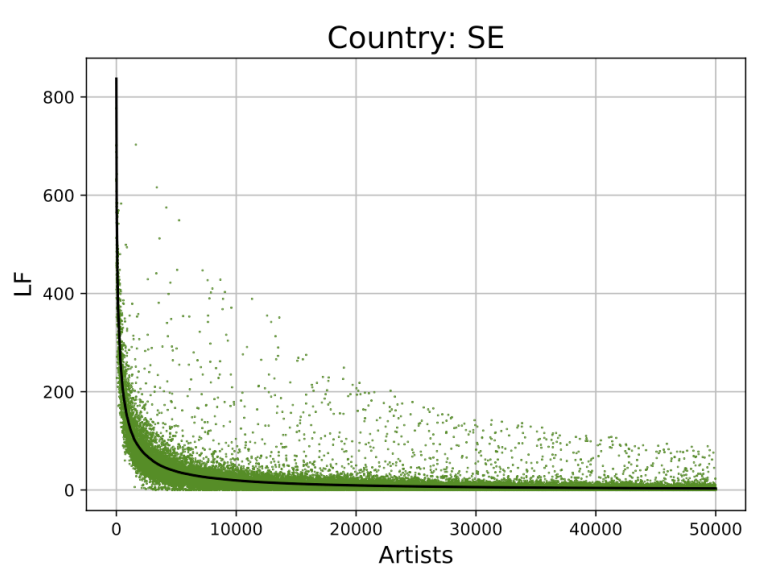

Figure 3. Listener frequency distribution over artists for Sweden

\subsection{Exemplary Study using Country-specific Recommendations}

Based on the results presented in Section 4.1, we now ask whether users may more satisfied when served with a state-of-the-art collaborative filtering MRS that tailors its recommendations based on nearest neighbors to users in the same country as compared to the one considering global mainstreaminess. We further analyze the influence of filtering the nearest neighbors with respect to the mainstreaminess group they belong to (Section 3.3).

Table 1 shows the RMSE for the global and country-specific mainstreaminess definitions and various levels of mainstreaminess, averaged over all considered countries, whereby RMSE is weighted by the number of users in the respective country. Results indicate an overall improvement (14.349 vs. 15.906) when using the country scope, considering all user sets (i.e., no differentiation whether high, mid, or low mainstreaminess). While there is a very slight fallback in RMSE for the high mainstreaminess user set (3.680 vs. 3.687), results show a considerable improvement for the mid set and even more for the low mainstreaminess user set. As it is particularly difficult to predict the preferences and listening behavior of the low segment, consisting of users with a specialized music taste, the low RMSE is remarkable.

Table 1. Weighted root mean square error (w.RMSE) for the global and country-specific mainstreaminess definitions and various levels of mainstreaminess, i.e. user sets, averaged over all considered countries

\begin{tabular}{|c|c|c|}
\hline measure & user set & w.RMSE \\
\hline \multirow{4}{*}{$R_{u, g}$} & all & 15.906 \\
\hline & high & 3.680 \\
\hline & mid & 7.443 \\
\hline & low & 19.183 \\
\hline \multirow{4}{*}{$R_{u, c}$} & all & 14.349 \\
\hline & high & 3.687 \\
\hline & mid & 4.270 \\
\hline & low & 3.692 \\
\hline
\end{tabular}

Table 2 shows the RMSE for the global and country-specific mainstreaminess definitions and various levels of mainstreaminess, for users of the exemplar countries United States, Finland, and Sweden, separately. Considering the entire country user set $(a l l)$, our results for these three countries indicate that the global mainstreaminess measure performs poorly for Finland (RMSE=27.084), a country far away from the global mainstream but particularly well for the United States (RMSE=5.327) and Sweden (RMSE=6.209), two countries highly oriented at the global mainstream. When tailoring recommendations based on the three levels of mainstreaminess separately, the accuracy achieved was overall better (or in some cases similar) compared to not considering the levels. Especially, the results for the US are remarkable since we can find for this country rather poor performance for the low mainstreaminess group, but good results for the mid and high groups, an observation which seems reasonable since it is in general "easier" for a MRS to suggest items to users whose taste is close to the mainstream. This observation holds for Finland and Sweden too, but the effect is much less pronounced. 
Tailoring recommendations to the country-specific mainstreaminess shows mixed results for the various user sets. Particularly for the United States, a global mainstream country, the measure performs quite poorly on a country-level without mainstreaminess level considered $(a l l)$, but very well when tailoring the recommendations to the user group with mainstreaminess similar to that of the target user (for all low, mid, and high segments). For Finland, the country with its distinct country-specific mainstream, the measure considering the country-specific mainstream performs well for all mainstreaminess levels, except for the mid one; however, considering all users in the country even outperforms categorizing users according to their mainstreaminess (RMSE $=3.976$ in country filtering versus $\mathrm{RMSE}=27.084$ on the global scope). For Sweden, results for all mainstreaminess segments are quite similar, and also similar to those realized on all Swedish users.

\section{Table 2. Root mean square error (RMSE) for the global and country-specific mainstreaminess definitions and various levels of mainstreaminess, i.e. user sets, for the United States, Finland, and Sweden}

\begin{tabular}{|c|c|c|c|}
\hline measure & country & user set & RMSE \\
\hline \multirow{12}{*}{$R_{u, g}$} & \multirow[t]{4}{*}{ US } & all & 5.327 \\
\hline & & high & 5.396 \\
\hline & & mid & 24.845 \\
\hline & & low & 28.544 \\
\hline & \multirow[t]{4}{*}{ FI } & all & 27.084 \\
\hline & & high & 3.909 \\
\hline & & mid & 4.135 \\
\hline & & low & 4.077 \\
\hline & \multirow[t]{4}{*}{ SE } & all & 6.209 \\
\hline & & high & 6.278 \\
\hline & & mid & 6.318 \\
\hline & & low & 6.436 \\
\hline \multirow{12}{*}{$R_{u, c}$} & \multirow[t]{4}{*}{ US } & all & 28.995 \\
\hline & & high & 5.360 \\
\hline & & mid & 5.411 \\
\hline & & low & 5.434 \\
\hline & \multirow[t]{4}{*}{ FI } & all & 3.976 \\
\hline & & high & 4.058 \\
\hline & & mid & 25.723 \\
\hline & & low & 4.085 \\
\hline & \multirow[t]{4}{*}{$\mathrm{SE}$} & all & 6.199 \\
\hline & & high & 6.225 \\
\hline & & mid & 6.473 \\
\hline & & low & 6.331 \\
\hline
\end{tabular}

\section{Discussion and Conclusion}

Investigated and discussed in various scientific disciplines, it is widely acknowledged that the assumption of culturally uniform Internet users does not reflect users' behavior in the real world in general. Making a similar assumption, in this paper we explored whether there are country-specific differences in users' listening behavior on music platforms (as reflected on the music platform Last.fm, based on 53,258 users from 47 countries).

The first main contribution of our work relates to country-specific differences in music listening behavior with respect to the degree of deviation from the global mainstream. Our results indicate that there are country-specific differences with respect to the most popular artists listened to in each country. While some countries are close to the global mainstream (e.g., the United States), other countries (e.g., Finland) show a distinct country-specific mainstream that is listened to in addition to the global mainstream. Another group of countries (e.g., Sweden) shows deviations from the global mainstream, having a lot of outliers in artist frequencies that do not reflect the global mainstream. At the same time, Sweden does, though, not show a clear Swedish mainstream that is listened to in parallel while ignoring the global mainstream; rather the global mainstream is important in the country but, still, users listen to some artists very frequently that are not part of the global mainstream. Thereby, it seems particularly interesting that the two countries, Finland and Sweden, which are geographically close to each other show such different country mainstreaminess profiles.

The second main contribution of our work relates to improvements for personalized music recommendations in terms of decreased rating prediction errors. In doing so, we compared tailoring music recommendations to three different mainstreaminess levels (low, mid, and high) in contrast to not considering these levels $(a l l)$. To this end, we also considered that the music mainstream may be defined from a global (as it is typically done) and from country-specific perspectives. Therefore, we employed two different mainstreaminess measurement approaches (global vs country-specific). This allowed us to study how the combination of a user's mainstreaminess and "country filtering" influence the quality of music recommendations. Our results suggest that such a combination works particularly well for countries far away from the global mainstream (e.g., Finland). For countries close to the global mainstream (e.g., United States), in contrast, the country-specific approach does not deliver satisfying results when applied to all users in the country, but it outperforms 
particularly for the low and mid mainstreaminess user set, compared to the global approach.

Being aware that the rating prediction experiments focused on only three rather dissimilar countries, our results suggest that tailoring music recommendations to a user's country may improve recommendation accuracy and, thus, the perceived recommendation quality of an MRS. Still, our results also indicate that the adopted measures do not perform equally well for all kinds of country mainstreaminess profiles. Consequently, it is important that an MRS also takes into account which country is addressed.

One avenue for further research is to develop advancements in mainstreaminess measurement that may further improve recommendation performance. Another avenue that could be taken would involve focusing on algorithmic advancements that may be described as "recommender of recommenders", meaning that depending on the identified user country and the respective country profile, different measurements and/or algorithms would be adopted for further steps in the recommendation process.

As another part of our future work, we will delve into detail for a larger scale of countries and will specifically analyze in which countries what kind of mainstreaminess functions perform particularly well or poorly. This may also form the basis for the above mentioned "recommender of recommenders" approach. Additional work will expand the perspective on cultural aspects. In this work, we used user country as a proxy. Future work on the role cultural aspects in MRS will take a more comprehensive perspective on culture, including various additional characteristics that shape a user's cultural background, such as religion or language.

Our findings presented in this paper have direct practical implications for music platforms that integrate MRS, including music streaming services such as Spotify and Pandora, but also multimedia platforms hosting music videos such as YouTube. Our presented approach can be readily adopted in realworld MRS. Theoretical implications relate to the existence of national boundaries on the global online market. This finding is particularly interesting as the music recording industry is considered a globally oriented market [24], compared to rather locally oriented markets (e.g., the market for food products [20]).

\section{Acknowledgments}

This research is supported by the Austrian Science Fund (FWF): P25655 and V579.

\section{References}

[1] Adomavicius, G., B. Mobasher, F. Ricci, and A. Tuzhilin, "Context-Aware Recommender Systems", AI Magazine, 32(3), 2011, pp. 67-80.

[2] Adomavicius, G., and A. Tuzhilin, "Toward the Next Generation of Recommender Systems: A Survey of the Stateof-the-Art and Possible Extensions", IEEE Transactions on Knowledge and Data Engineering, 17(6), 2005, pp. 734-749.

[3] Ahn, H.J., "Utilizing Popularity Characteristics for Product Recommendation", International Journal of Electronic Commerce, 11(2), 2006, pp. 59-80.

[4] Anderson, C., "The Long Tail", Wired Magazine, 10 Jan, 2004, https://www.wired.com/2004/10/tail/, accessed 17 Sept 2017.

[5] Anderson, C., The Long Tail: Why the Future of Business Is Selling Less of More, Hyperion, New York, NY, 2006.

[6] Baek, Y.M., "Relationship between Cultural Distance and Cross-Cultural Music Video Consumption on Youtube", Social Science Computer Review, 33(6), 2015, pp. 730-748.

[7] Bauer, C., M. Kholodylo, and C. Strauss, "Music Recommender Systems: Challenges and Opportunities for Non-Superstar Artists", 30th Bled eConference, 2017, pp. 2132.

[8] Bauer, C., and A. Novotny, "A Consolidated View of Context for Intelligent Systems", Journal of Ambient Intelligence and Smart Environments, 9(4), 2017, pp. 377393.

[9] Bawden, D., and L. Robinson, "The Dark Side of Information: Overload, Anxiety and Other Paradoxes and Pathologies", Journal of Information Science, 35(2), 2009, pp. 180-191

[10] Boer, D., R. Fischer, M. Strack, M.H. Bond, E. Lo, and J. Lam, "How Shared Preferences in Music Create Bonds between People: Values as the Missing Link", Personality and Social Psychology Bulletin, 37(9), 2011,pp. 1159-1171.

[11] Bogdanov, D., M. Haro, F. Fuhrmann, A. Xambo, E. Gomez, and P. Herrera, "Semantic Audio Content-Based Music Recommendation and Visualization Based on User Preference Examples", Information Processing \& Management, 49(1), 2013, pp. 13-33.

[12] Bogdanov, D., and P. Herrera, "How Much Metadata Do We Need in Music Recommendation?: A Subjective Evaluation Using Preference Sets", 12th International Conference on Music Information Retrieval (ISMIR), 2011, pp. 97-102.

[13] Bonneville-Roussy, A., P.J. Rentfrow, M.K. Xu, and J. Potter, "Music through the Ages: Trends in Musical Engagement and Preferences from Adolescence through Middle Adulthood", Journal of Personality and Social Psychology, 105(4), 2013, pp. 703-717. 
[14] Brown, R.A., "Music Preferences and Personality among Japanese University Students", International Journal of Psychology, 47(4), 2012, pp. 259-268.

[15] Budzinski, O., and J. Pannicke, "Do Preferences for Pop Music Converge across Countries?: empirical Evidence from the Eurovision Song Contest", Creative Industries Journal, 10(2), 2017, pp. 168-187.

[16] Casey, M.A., R. Veltkamp, M. Goto, M. Leman, C. Rhodes, and M. Slaney, "Content-Based Music Information Retrieval: Current Directions and Future Challenges", Proceedings of the IEEE, 96(4), 2008, pp. 668-696.

[17] Celma, Ò., Music Recommendation and Discovery: The Long Tail, Long Fail, and Long Play in the Digital Music Space, Springer, Berlin, Heidelberg, Germany, 2010.

[18] Celma, Ò., and P. Cano, "From Hits to Niches?: Or How Popular Artists Can Bias Music Recommendation and Discovery", 2nd KDD Workshop on Large-Scale Recommender Systems and the Netflix Prize Competition (NETFLIX), 2008.

[19] Cheng, Z., and J. Shen, "Just-for-Me: An Adaptive Personalization System for Location-Aware Social Music Recommendation", ACM International Conference on Multimedia Retrieval (ICMR), 2014, pp. 185-194.

[20] Cleveland, M., J.I. Rojas-Méndez, M. Laroche, and N. Papadopulos, "Identity, Culture, Dispositions and Behavior: A Cross-National Examination of Globalization and Culture Change", Journal of Business Research, 69(3), 2016, pp. 1090-1102.

[21] Colley, A., "Young People's Musical Taste: Relationship with Gender and Gender-Related Traits", Journal of Applied Social Psychology, 38(8), 2008, pp. 2039-2055.

[22] Cremonesi, P., F. Garzotto, R. Pagano, and M. Quadrana, "Recommending without Short Head", 23rd International Conference on World Wide Web (WWW), 2014, pp. 245-246.

[23] Dey, A.K., and G.D. Abowd, "Towards a Better Understanding of Context and Context-Awareness", Workshop on The What, Who, Where, When, and How of Context-Awareness, part of CHI, 2000.

[24] Dolata, U., The Transformative Capacity of New Technologies: A Theory of Sociotechnical Change, Routledge, London, United Kindom, 2013.

[25] Green, S.J., P. Lamere, J. Alexander, F. Maillet, S. Kirk, J. Holt, J. Bourque, and X.W. Mak, "Generating Transparent, Steerable Recommendations from Textual Descriptions of Items", 3rd ACM Conference on Recommender Systems (RecSys), 2009, pp. 281-284.

[26] Hracs, B.J., M. Seman, and T.E. Virani (eds.), The Production and Consumption of Music in the Digital Age, Routledge, New York, NY, 2017.

[27] Jones, M.L., "Hofstede - Culturally Questionable?", Oxford Business \& Economics Conference, 2007.
[28] Koren, Y., and R. Bell, "Advances in Collaborative Filtering", in Ricci, F., L. Rokach, B. Shapira, and P.B. Kantor, (eds.): Recommender Systems Handbook, 2nd edn., Springer, New York, NY, 2015, pp. 77-118.

[29] Krismayer, T., M. Schedl, P. Knees, and R. Rabiser, "Prediction of User Demographics from Music Listening Habits", 15th International Workshop on Content-based Multimedia Indexing (CBMI), 2017.

[30] Laplante, A., "Improving Music Recommender Systems: What Can We Learn from Research on Music Tags?", 15th International Society for Music Information Retrieval Conference (ISMIR), 2014, pp. 451-456.

[31] Lee, H.H., and X. Hu, "Cross-Cultural Similarities and Differences in Music Mood Perception", iConference 2014, 2014, pp. 249-269.

[32] Levy, M., and M. Sandler, "Learning Latent Semantic Models for Music from Social Tags", Journal of New Music Research, 37(2), 2008, pp. 137-150.

[33] Li, S., and E. Karahanna, "Peer-Based Recommendations in Online B2c E-Commerce: Comparing Collaborative Personalization and Social Network-Based Personalization", 45th Hawaii International Conference on System Sciences (HICSS), 2012, pp. 733-742.

[34] Linden, G., B. Smith, and J. York, "Amazon.Com Recommendations: Item-to-Item Collaborative Filtering", IEEE Internet Computing, 7(1), 2003, pp. 76-80.

[35] Ma, H., T.C. Zhou, M.R. Lyu, and I. King, "Improving Recommender Systems by Incorporating Social Contextual Information", ACM Transactions on Information Systems, 29(2), 2011,

[36] Magno, T., and C. Sable, "A Comparison of SignalBased Music Recommendation to Genre Labels, Collaborative Filtering, Musicological Analysis, Human Recommendation, and Random Baseline", 9th International Conference on Music Information Retrieval (ISMIR), 2008, pp. 161-166.

[37] McSweeney, B., "Hofstede's Model of National Cultural Differences and Their Consequences: A Triumph of Faith - a Failure of Analysis", Human Relations, 55(1), 2002, pp. 89118 .

[38] Montaner, M., B. López, and J.L. de la Rosa, "A Taxonomy of Recommender Agents on the Internet", Artificial Intelligence Review, 19(4), 2003, pp. 285-330.

[39] Morrison, S.J., and S.M. Demorest, "Cultural Constraints on Music Perception and Cognition", Progress in brain research, 178, 2009, pp. 67-77.

[40] Oechslein, O., and T. Hess, "The Value of a Recommendation: The Role of Social Ties in Social Recommender Systems", 47th Hawaii International Conference on System Science (HICSS), 2014, pp. 18641873. 
[41] Oestreicher-Singer, G., and A. Sundararajan, "Recommendation Networks and the Long Tail of Electronic Commerce", MIS Quarterly, 36(1), 2012, pp. 65-83.

[42] Power, D., and D. Hallencreutz, "Competitiveness, Local Production Systems and Global Commodity Chains in the Music Industry: Entering the Us Market", Regional Studies, 41(3)pp. 377-389.

[43] Rentfrow, P.J., and S.D. Gosling, "The Do Re Mi's of Everyday Life: The Structure and Personality Correlates of Music Preferences", Journal of Personality and Social Psychology, 84(6), 2003, pp. 1236-1256.

[44] Ricci, F., L. Rokach, and B. Shapira (eds.), Recommender Systems Handbook, Springer, 2nd edn, New York, NY, 2015.

[45] Rutten, P., "Local Popular Music on the National and International Markets", Cultural Studies, 5(3), 1991, pp. 294305 .

[46] Salakhutdinov, R., and A. Mnih, "Probabilistic Matrix Factorization", 21st Annual Conference on Neural Information Processing Systems (NIPS), 2007, pp. 12571264.

[47] Schedl, M., "Ameliorating Music Recommendation: Integrating Music Content, Music Context, and User Context for Improved Music Retrieval and Recommendation", International Conference on Advances in Mobile Computing \& Multimedia (MoMM), 2013.

[48] Schedl, M., "The Lfm-1b Dataset for Music Retrieval and Recommendation", ACM International Conference on Multimedia Retrieval (ICMR), 2016, pp. 103-110

[49] Schedl, M., and C. Bauer, "Distance- and Rank-Based Music Mainstreaminess Measurement", 25th International Conference on User Modeling, Adaptation and Personalization (UMAP): 2nd Workshop on Surprise, Opposition, and Obstruction in Adaptive and Personalized Systems (SOAP), 2017, pp. 364-367.

[50] Schedl, M., E. Gómez, and J. Urbano, "Music Information Retrieval: Recent Developments and Applications", Foundations and Trends in Information Retrieval, 8(2-3), 2014, pp. 127-261.

[51] Schedl, M., and D. Hauger, "Tailoring Music Recommendations to Users by Considering Diversity, Mainstreaminess, and Novelty", 38th International ACM Conference on Research and Development in Information Retrieval (SIGIR), 2015, pp. 947-950.

[52] Schedl, M., D. Hauger, K. Farrahi, and M. Tkalčič, "On the Influence of User Characteristics on Music Recommendation Algorithms", 37th European Conference on Information Retrieval (ECIR), 2015, pp. 339-345.

[53] Schedl, M., P. Knees, B. McFee, D. Bogdanov, and M. Kaminskas, "Music Recommender Systems", in Ricci, F., L. Rokach, B. Shapira, and P.B. Kantor, (eds.): Recommender
Systems Handbook, 2nd edn., Springer, New York, NY, 2015, pp. 453-492.

[54] Schedl, M., T. Pohle, P. Knees, and G. Widmer, "Exploring the Music Similarity Space on the Web", ACM Transactions on Information Systems, 29(3), 2011,

[55] Schedl, M., and M. Tkalčič, "Genre-Based Analysis of Social Media Data on Music Listening Behavior", 1st ACM International Workshop on Internet-Scale Multimedia Management (ISMM), 2014, pp. 9-13.

[56] Singhi, A., and D.G. Brown, "On Cultural, Textual and Experiential Aspects of Music Mood", 5th International Society for Music Information Retrieval Conference (ISMIR), 2014.

[57] Slaney, M., "Web-Scale Multimedia Analysis: Does Content Matter?", IEEE Multimedia, 18(2), 2011, pp. 12-15.

[58] Stevens, C.J., "Music Perception and Cognition: A Review of Recent Cross-Cultural Research", Topics in Cognitive Science, 4(4), 2012, pp. 653-667.

[59] ter Bogt, T.F.M., M.J.M.H. Delsing, M. van Zalk, P.G. Christenson, and W.H.J. Meeus, "Intergenerational Continuity of Taste: Parental and Adolescent Music Preferences", Social Forces, 90(1), 2011, pp. 297-319.

[60] Turnbull, D.R., L. Barrington, G. Lanckriet, and M. Yazdani, "Combining Audio Content and Social Context for Semantic Music Discovery", 32nd international ACM SIGIR conference on Research and development in information retrieval (SIGIR), 2009, pp. 387-394.

[61] Vigliensoni, G., and I. Fujinaga, "Automatic Music Recommendation Systems: Do Demographic, Profiling, and Contextual Features Improve Their Performance?", 17th International Society for Music Information Retrieval Conference (ISMIR), 2016, pp. 94-100.

[62] Wang, J., Y. Liu, and J. Sun, "Diversified Recommendation Incorporating Item Content Information Based on Moea/D", 49th Hawaii International Conference on System Sciences (HICSS), 2016, pp. 688-696.

[63] Wang, X., D. Rosenblum, and Y. Wang, "ContextAware Mobile Music Recommendation for Daily Activities", 20th ACM Conference on Multimedia (MM), 2012, pp. 99108.

[64] Yan, Y., T. Liu, and Z. Wang, "A Music Recommendation Algorithm Based on Hybrid Collaborative Filtering Technique", 5th National Conference on Social Media Processing (SMP), 2015, pp. 233-240.

[65] Yang, J.A., "Effects of Popularity-Based News Recommendations ("Most-Viewed") on Users' Exposure to on-Line News", Media Psychology, 19(2), 2016, pp. 243271. 